\title{
Percepción del perfil profesional de los fisioterapeutas de una universidad pública
}

\section{Perception of the professional profile of the physiotherapist of a public university}

\author{
Jessica López-Laverde'; Myriam Maya-Valencia ${ }^{1}$; Cristian-D Palacio-Ruiz ${ }^{1}$
}

\begin{abstract}
Forma de citar: López Laverde J, Maya Valencia M, Palacio Ruiz CD. Percepción del perfil profesional de los fisioterapeutas de una universidad pública. Rev Univ Ind Santander Salud. 2019; 51(2): 129-134. doi: http://dx.doi.org/10.18273/revsal.v51n2-2019005 (c) (1)
\end{abstract}

\section{Resumen}

Introducción: El perfil profesional consiste en la descripción de las habilidades, conocimiento y destrezas que un profesional debe tener o tiene para ejercer eficientemente en un puesto de trabajo; son actividades que los jefes directivos esperan que asuma el trabajador como parte del desarrollo de su profesión. Objetivo: Describir la percepción del perfil profesional del egresado de fisioterapia en una Institución Universitaria Pública desde la perspectiva de los jefes directos. Metodología: Estudio descriptivo transversal, con una muestra de 25 jefes directos de los egresados de una universidad pública, seleccionados aleatoriamente de la base de datos de egresados de la institución; quienes diligenciaron un cuestionario en físico de 33 preguntas, elaborado por los investigadores. Resultados: Los cuestionarios fueron analizados con Microsoft Excel. De acuerdo con la valoración dada por los jefes directos del fisioterapeuta, se caracterizó por un alto grado de compromiso profesional y ético, conocimientos teóricos y prácticos, además de la calidad en el trabajo y cumplimiento. Sin embargo, la competencia menor calificada, fue el conocimiento de idiomas. Conclusiones: La percepción del perfil profesional para el jefe directo, tiene que ver con las competencias específicas y con la práctica basada en la evidencia, siendo las que obtuvieron un valor de $80 \%$, por debajo del resultado obtenido en las competencias genéricas en el perfil observado en el profesional de la institución superior.

Palabras Clave: Perfil profesional; Competencias; Jefes directos; Fisioterapia.

\begin{abstract}
Introduction: Professional profile involves the description of abilities, knowledge and skills that every professional has or should have for an efficient work performance. This entails activities that executive heads expect that workers are in charge as part of their professional development. Objective: To describe the perception of the professional profile of the physiotherapy graduate in a public university institution from the perspective of the direct heads. Methodology: Cross-sectional descriptive study with a sample of 25 direct heads of graduates of a public university, randomly selected from the database of graduates of the institution. The participants answered

1. Institución Universitaria Escuela Nacional del Deporte. Cali, Colombia.

Correspondencia: Jessica López Laverde. Dirección: Calle 9 \#34-01, Cali. Teléfono: +57 3176385957. Correo electrónico: jessica.lopez@, endeporte.edu.co
\end{abstract}


a 33-question printed questionnaire designed by the researchers. Results: Every questionnaire was analyzed with Microsoft Excel. In accordance to the given assessment of the direct heads, the physiotherapists were characterized by having high professional and ethical commitment, theoretical and practical insights, and high quality in work performance and compliance at work. Nevertheless, the competence with the lowest score was the knowledge on foreign languages. Conclusions: The perception of the professional profile from the direct heads has to do with the specific competences and the evidence-based practice. These obtained a value of $80 \%$, below the result obtained in the generic competences in the profile observed in the professional of the higher institution.

Keywords: Professional profile; Competences; Direct heads; Physiotherapy.

\section{Introducción}

Las competencias profesionales en un estudiante son el conjunto de acciones que éste realiza cuando interactúa significativamente en el contexto donde desarrolla la práctica profesional, por lo cual resulta importante considerar la definición de competencia, que se resume como un saber hacer en contexto ${ }^{1}$. Mientras que las competencias en el profesional graduado, se relacionan en el ejercicio de su desempeño laboral, las cuales una vez obtenidas se expresan como el dominio que se tiene en la integración y desenvolvimiento del saber y hacer en el ejercicio laboral. En este sentido, se considera el perfil profesional como un "conjunto de rasgos y capacidades que, certificadas apropiadamente por quien tiene la competencia jurídica para ello, permiten que alguien sea reconocido por la sociedad como tal profesional, pudiéndole encomendar tareas para las que se le supone capacitado y competente ${ }^{2}$.

Del mismo modo, las competencias de acuerdo con la Organización para la Cooperación y el Desarrollo Económicos (OCDE) en 2014, están conformadas por conocimientos y destrezas, que incluyen la habilidad para enfrentar demandas complejas, apoyándose y movilizando destrezas y actitudes en un contexto particular ${ }^{3}$. Estas competencias se constituyen en una ganancia asociada a la apropiación de una cadena de saberes, como son: conocimientos, habilidades, valores, actitudes, emociones ${ }^{1}$. Con el fin de lograr alcanzar o desplegar una competencia, se debe relacionar y asegurar siempre una serie de saberes asociados a esta e igualmente se requiere estar al tanto para qué son y la manera adecuada de ejecutarlas. Para esto, es necesario establecer que desde la academia se deben elegir los contenidos curriculares adecuados para trabajar y desarrollar las competencias que puedan dar fe del egresado que sacará a nivel ocupacional.

De este modo, el concepto sobre perfil profesional, consiste en la descripción de las habilidades que un futuro profesional debe tener (o tiene) para ejercer eficientemente en un puesto de trabajo ${ }^{4}$. Es por esto que, se debe determinar lo que un jefe directo esperaría que asuma un profesional, considerando el contexto donde se ejecuta la profesión, los campos de desempeño, su impacto en la comunidad, que tenga un adecuado sustento académico, científico y tecnológico ${ }^{5}$.

Por lo anterior, es importante estimar el documento que sobre las competencias del fisioterapeuta en Colombia ha realizado ASCOFI y ASCOFAFI ${ }^{6}$, en donde se dividen las competencias transversales y específicas, algunas de las cuales fueron consideradas en este estudio. Partiendo de dichos lineamientos, es que se desarrolla el consenso de la formación y práctica de los programas de fisioterapia. Por tanto, es significativo estimar la percepción del jefe directo, quien es la persona más cercana en conocer la formación profesional que exhibe un fisioterapeuta en correspondencia con su ejercicio laboral.

Siendo el objetivo del presente trabajo, determinar la percepción del perfil profesional del fisioterapeuta de una institución universitaria pública desde la perspectiva de los jefes directos.

\section{Metodología}

El tipo de estudio fue un descriptivo observacional, la unidad de análisis los jefes directos de los egresados de fisioterapia de la Institución Superior, tomados de la base de datos del programa de los años 2003 al 2017 de manera aleatoria. El estudio se realizó en tres fases: una de las cuales fue la revisión de literatura, otra donde se elaboró un cuestionario, con base en las competencias profesionales del fisioterapeuta en Colombia propuestas por el Ministerio de Salud y Protección Social de Colombia en el trabajo "Perfil Profesional y Competencias del Fisioterapeuta en Colombia", del cual se tomaron 19 competencias que describen el perfil profesiográfico en los campos de desempeño. Este cuestionario se dividió en dos partes, una de las cuales contenía nueve preguntas, con información sociodemográfica del cuestionario y del campo laboral que permitió dar respuesta al primer 
objetivo. La segunda parte del cuestionario, incluyó una tabla compuesta con 19 competencias seleccionadas del perfil profesional ${ }^{6}$, que fueron valoradas en dos secciones por los jefes directos: en una sección se estimó la importancia de estas competencias para estos, respecto los procesos de valoración del ejercicio laboral que su organización ha verificado en el profesional de fisioterapia y en la siguiente sección, el cumplimiento de estas competencias en el fisioterapeuta de la Institución Superior pública. Para la valoración de cada competencia, se aplicó la escala de Likert de 1 a 5, donde 1 equivalió a estar "muy en desacuerdo", el 2 significó "en desacuerdo", el 3 "ni de acuerdo, ni en desacuerdo", el 4 representó un "de acuerdo" y finalmente el valor 5 correspondió a "muy de acuerdo".

Y una última fase, que consistió en someter el cuestionario anterior a revisión por parte de un grupo de docentes del grupo de investigación del consejo académico de la institución superior. Posteriormente se llevó a cabo la prueba piloto, con el fin de determinar la construcción y claridad del cuestionario, con base en los hallazgos se realizaron los ajustes pertinentes, como la necesidad de acompañamiento por parte de una persona del grupo investigador en la aplicación del cuestionario. Finalmente, en el análisis de los resultados, se caracterizó a las empresas de los jefes directos, de acuerdo a la naturaleza jurídica, número de empleados fisioterapeutas y fisioterapeutas graduados de la institución superior, mediante la aplicación de tablas de frecuencia. Así mismo, en estas tablas se representó cada competencia del cuestionario, las cuales fueron codificadas numéricamente, para facilitar la lectura de acuerdo al valor de la escala de Likert, lo que permitió describir las competencias profesionales que los jefes directos consideraron pertinentes y que caracterizaban al profesional de la institución superior, para esto se compiló en un porcentaje la suma de la competencia que obtuvo un valor entre 4 y 5 dado por la escala de Likert, de tal manera que las respuestas en estas categorías fueron las que indicaron mayor aceptación por parte de los jefes directos de acuerdo al grupo investigador, siendo sobreestimadas respecto a las que indicaron menor valor. Para de aquí proceder a elaborar las respectivas tablas de frecuencias. Finalmente, los resultados de la respuesta de las competencias de la Tabla 4. fueron agrupadas de acuerdo con el resultado de la suma de los porcentajes, en orden descendente.

\section{Resultados}

A enero de 2017 la institución superior contaba con 1610 graduados, reportados en la base de datos, de donde se tomaron 25 entidades aleatoriamente, las cuales contaban con 521 fisioterapeutas empleados, de estos $167(32 \%)$ correspondían a graduados de la institución en estudio; así mismo de las entidades participantes, $16(64 \%)$ empresas pertenecían al sector privado, 8 (32\%) públicas y $1(4 \%)$ mixta. La distribución de las entidades, de acuerdo al nivel de complejidad correspondió al (28\%) para el Nivel III y (72\%) para el nivel I y II.

\section{Características sociodemográficas de los jefes directos}

Todos los jefes directos fueron de nacionalidad colombiana, nueve de ellos de género masculino y 16 femenino, así mismo, los cargos desempeñados fueron 16 coordinadores, seis gerentes y tres tenían otro tipo de cargo, distribuidos en las siguientes profesiones: 17 fisioterapeutas, cinco administradores, dos profesionales del deporte y una enfermera.

La institución de educación superior de egreso de los jefes directos, estuvo representada en $10(40 \%)$ instituciones públicas y 15 (60\%) privadas, (Tabla 1).

Tabla 1. Institución de educación superior de egreso del jefe directo.

\begin{tabular}{lcc}
\hline $\begin{array}{c}\text { Institución de educación superior } \\
\text { de egreso del jefe directo }\end{array}$ & Frecuencia & Porcentaje \\
\hline IU Escuela Nacional de Deporte & 5 & 20,0 \\
U del Valle & 5 & 20,0 \\
U Santiago de Cali & 3 & 12,0 \\
FU María Cano & 2 & 8,0 \\
Otras & 10 & 40,0 \\
Total & 25 & 100,0 \\
\hline
\end{tabular}

De acuerdo con la actividad económica, las empresas en su mayoría fueron prestadoras de servicios de salud 22 (88\%), seguida de 3 (12\%), que ofrecían atención en salud pública y administración de manera conjunta.

(Tabla. 2)

Tabla 2. Campo acción fisioterapeuta de la institución superior.

\begin{tabular}{lcc}
\hline $\begin{array}{c}\text { Campo acción del } \\
\text { Fisioterapeuta de la Institución }\end{array}$ & Frecuencia & Porcentaje \\
\hline Clínico & 16 & 64,0 \\
Deportivo & 4 & 16,0 \\
Laboral & 2 & 8,0 \\
Mixta & 3 & 12,0 \\
Total & 25 & 100,0 \\
\hline
\end{tabular}

A continuación, se describen las competencias que los jefes directos valoraron en el fisioterapeuta de la institución superior, como: conocimientos, habilidades, 
actitudes y valores, las cuales fueron agrupadas en valor porcentual en orden descendente de los resultados: "de acuerdo" o "muy de acuerdo" como fue:

Tabla 3. Competencias observadas en el fisioterapeuta de la institución superior.

\begin{tabular}{|c|c|c|}
\hline Competencias & Frecuencia & Porcentaje \\
\hline Presentación personal & 25 & $100 \%$ \\
\hline Relaciones interpersonales & 23 & $92 \%$ \\
\hline $\begin{array}{l}\text { Capacidad de trabajo en equipo y } \\
\text { Calidad de trabajo }\end{array}$ & 22 & $88 \%$ \\
\hline $\begin{array}{l}\text { Cumplimiento, habilidad - } \\
\text { rendimiento y conocimientos } \\
\text { prácticos }\end{array}$ & 21 & $84 \%$ \\
\hline $\begin{array}{l}\text { Conocimientos teóricos, manejo } \\
\text { de herramientas informáticas, } \\
\text { capacidad de aprendizaje, } \\
\text { capacidad creativa e innovadora, } \\
\text { práctica basada en evidencia } \\
\text { científica y actualización de } \\
\text { políticas y procedimientos }\end{array}$ & 20 & $80 \%$ \\
\hline $\begin{array}{l}\text { Capacidad de liderazgo, } \\
\text { compromiso ético, profesional, } \\
\text { capacidad de análisis - evaluación } \\
\text { de problemas, capacidad de } \\
\text { comunicación oral y escrita }\end{array}$ & 19 & $76 \%$ \\
\hline Iniciativa & 18 & $72 \%$ \\
\hline Conocimientos de otros idiomas & 6 & $24 \%$ \\
\hline
\end{tabular}

Las competencias que los jefes directos consideran deben tener un fisioterapeuta en una organización laboral.

Tabla 4. Competencias requeridas por los jefes directos en un fisioterapeuta.

\begin{tabular}{|c|c|c|}
\hline Competencias & Frecuencia & Porcentaje \\
\hline $\begin{array}{l}\text { Presentación } \\
\text { comunicación oral y escrita, } \\
\text { conocimientos prácticos, práctica } \\
\text { basada en evidencia científica, } \\
\text { actualización de políticas y } \\
\text { procedimientos, creatividad } \\
\text { e innovación, habilidad y } \\
\text { rendimiento, calidad del trabajo }\end{array}$ & 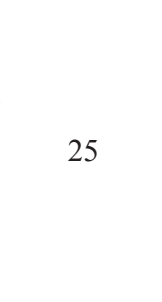 & $100 \%$ \\
\hline $\begin{array}{l}\text { Compromiso profesional, ético y } \\
\text { capacidad de aprendizaje }\end{array}$ & 24 & $96 \%$ \\
\hline $\begin{array}{l}\text { Manejo de herramientas } \\
\text { informáticas y trabajo en equipo }\end{array}$ & 21 & $84 \%$ \\
\hline Conocimiento teórico & 20 & $80 \%$ \\
\hline $\begin{array}{l}\text { Análisis y evaluación de problemas } \\
\text { y cumplimiento }\end{array}$ & 19 & $76 \%$ \\
\hline $\begin{array}{l}\text { Capacidad de liderazgo y relaciones } \\
\text { interpersonales }\end{array}$ & 15 & $60 \%$ \\
\hline Iniciativa & 14 & $56 \%$ \\
\hline Conocimientos de otros idiomas & 12 & $48 \%$ \\
\hline
\end{tabular}

\section{Discusión}

Se encontró que los jefes directos perciben que el egresado de la institución superior cuenta con una formación adecuada de las competencias genéricas como son el cuidado de la imagen corporal, relaciones interpersonales, capacidad de trabajo en equipo, la habilidad y rendimiento, calidad del trabajo y conocimientos prácticos. Así mismo, los jefes directos no consideran estas competencias genéricas como las más valoradas y esperarían tuviera un fisioterapeuta a su criterio en la ejecución de sus labores. Sin embargo, los encuestados manifiestan estar muy de acuerdo en que las competencias como comunicación oral y escrita, práctica basada en la evidencia, actualización de políticas, creatividad e innovación, compromiso ético y capacidad de aprendizaje son muy importantes para el buen desempeño laboral de un fisioterapeuta. No obstante, hay coincidencias en competencias como conocimientos prácticos, habilidad - rendimiento y calidad de trabajo. De manera análoga, competencias como manejo de herramientas informáticas y trabajo en equipo fueron estimadas como altamente valoradas en lo que ven en el fisioterapeuta de la institución superior y lo que esperarían en un profesional.

Siendo estos resultados distintos, al descrito en el informe final del Proyecto Tuning en América Latina del 2004 al $2007^{7}$, el cual consideró que las competencias como liderazgo y trabajo en equipo, no aparecen en la percepción del documento por parte de los empleadores, por cuanto estos últimos dan por manifiestas estas competencias en el estudio de pregrado. De otro lado un estudio de Cabeza, et al. ${ }^{8}$ en el 2017, estableció que las competencias genéricas con más relevancia en las seis regiones de Colombia para empleadores y graduados son: capacidad para identificar planear y resolver problemas; capacidad de tomar decisiones y compromiso con la calidad. Otros datos similares al anterior, arrojó el estudio realizado por Cabrera, et al. ${ }^{9}$ destacando que los empleadores valoran principalmente las competencias genéricas, concretamente interpersonales (entre las más valoradas, el trabajo en equipo) y sistémicas (el liderazgo) y, en menor medida, las instrumentales. No obstante, el estudio de la AQU (Agència per a la Qualitat del Sistema Universitari de Catalunya $)^{10}$ encontró que dentro de una escala de 0 a 10, las competencias que los empleadores de la muestra consideraron más importantes fueron la responsabilidad en el trabajo $(8,9)$, la capacidad de aprender $(8,4)$ y el trabajo en equipo $(8,3)$. Las menos importantes fueron las habilidades de negociación $(6,4)$ y el liderazgo $(6,6)$ 
Por lo anterior, es importante tener en cuenta que las competencias genéricas (instrumentales, interpersonales y sistémicas) aunque demarcan las competencias básicas en la profesión, deben ser explicitas en el profesional con el fin de que sean puestas en el contexto laboral, de ahí que el estudio de Aurelio, et al. ${ }^{11}$ determinó que este tipo de competencias satisfacen requerimientos personales, relacionados con la ética y la percepción de triunfo en la vida y con el ambiente social. Muy similar a los resultados de Muñoz, et al. ${ }^{12}$, quienes evidenciaron que todas las competencias interpersonales y sistémicas analizadas se clasificaron como fortalezas percibidas por los empleadores y las que más cerca están de cubrir las expectativas, después de lo ético. Semejante al estudio realizado por Peterson y Roca ${ }^{13}$, quienes encontraron que "los egresados desde la perspectiva del empleador están capacitados en habilidades y competencias básicas para su desempeño profesional, especificamente en responsabilidad social y ética, disciplina, organización y trabajo en equipo, para satisfacer las necesidades del medio con un buen desempeño. Lo que podría llevar a confirmar que los jefes directos parten del hecho, que un egresado debe poseer estas competencias básicas genéricas como parte básica del quehacer laboral.

Mientras que la valoración para las competencias específicas en el profesional de la institución superior, como las destrezas que influyen en la productividad y la capacidad para realizar una intervención con el método más adecuado, estuvieron calificadas en un $84 \%$, siendo consideradas por los jefes directos como fundamentales en el desarrollo de la profesión, además de encontrarlas dinámicas en cuanto al saber hacer acorde a la labor del fisioterapeuta. En este sentido, Bernal ${ }^{14}$, considera que la determinación de los conocimientos de un dominio disciplinar ocurre en el momento en que se detallan las competencias profesionales específicas.

Agregando a lo anterior, en el presente estudio lo que suponen los jefes directos debiera tener un profesional de fisioterapia, dentro de las competencias específicas fue: calidad del trabajo, como resultado final alcanzado en los trabajos realizados, conocimientos prácticos $84 \%$, la práctica basada en la evidencia científica $80 \%$, que garantice que los procesos realizados sobre la población intervenida se basa en la mejor evidencia disponible, igualmente actualización de políticas y procedimientos $80 \%$, que define la buena práctica en fisioterapia ${ }^{3}$ y asegura que los servicios profesionales que prestan, cumplen a cabalidad con todos ellos. Agregado a lo anterior, dentro de las genéricas, se halló la creatividad e innovación 100\%, como la capacidad demostrada para aportar ideas y soluciones que contribuyan creativamente en la dinámica de la organización, igualmente con el mismo valor la habilidad para describir información identificada según sus resultados. Estas observaciones son similares al estudio de Dussán, et al. ${ }^{15}$, que permitió tener en cuenta que "En el Caribe las competencias coincidentes son: capacidad de aplicar los conocimientos en la práctica, capacidad para tomar decisiones, compromiso con la calidad, ejercer el liderazgo para el logro y consecución de metas en la organización esto es el $40 \%$ de las competencias requeridas". Mientras que el estudio de Villabona, et al. ${ }^{16}$, mostró que una de las particularidades del egresado de fisioterapia de la Universidad Industrial de Santander, que obtuvo uno de los promedios más bajos $3.6 \pm 9$, fue el aspecto relacionado el desarrollo político, social, cultural y económico del país.

En otro orden de ideas, el presente estudio demostró también, que los jefes directos establecieron la ponderación más baja $48 \%$, para conocimientos de otros idiomas. Sin embargo, vale la pena destacar que para los jefes directos, existe una ponderación alta en lo que se espera y tiene el fisioterapeuta de la institución superior, como es la competencia en relación con los conocimientos prácticos. Mientras que la iniciativa, no fue tan importante para los jefes directos, lo que podría estar relacionado con la hegemonía que le dan al saber y al saber hacer, en donde prevaleció el saber, como una herramienta primordial en el momento de contratar a un graduado, esto está relacionado con el estudio de Sánchez, et al. ${ }^{17}$, que mostró que el $48.69 \%$ de los empleadores entrevistados lo califican de importante y $39.74 \%$ de muy importante, sumando un $88.43 \%$.

Finalmente, la percepción del fisioterapeuta de una Institución Superior por parte de los jefes directos, es que se encuentran preparados en las competencias genéricas básicas como relaciones interpersonales, capacidad de trabajo en equipo y calidad de trabajo, entre otras, ahora bien, observan que se debe fortalecer en estos: la capacidad de análisis y la práctica basada en la evidencia.

\section{Conclusiones}

Se concluye que los jefes directos estiman en un fisioterapeuta, las competencias específicas más que las genéricas, las cuales consideran son las afines al perfil profesional laboral, que debieran ser la fundamentación en el lineamiento de una malla curricular. Así mismo, refieren que dentro de las competencias genéricas primordiales, el compromiso profesional y ético, son 
de las más importantes, pues distinguen que de aquí se desprende la confiabilidad y entrega de un profesional de fisioterapia.

En contraste, algunos jefes directos no valoran como importante la competencia en el conocimiento de otros idiomas, a diferencia de los jefes que pertenecen a entidades que cuentan con certificación en calidad, los cuales requieren el cumplimiento de esta competencia.

Al ser el profesional de fisioterapia de la institución superior, el puente entre la academia y el mundo laboral, surge la necesidad de continuar realizando investigaciones sobre el perfil profesional, en donde la unidad de análisis sea el egresado además del empleador, con el fin de que sirva de insumo en la toma de decisiones sobre el plan de estudio del programa.

\section{Agradecimientos}

A la institución de educación superior, al grupo de investigación de estudios en salud y sociedad y al programa de fisioterapia.

\section{Consideraciones Éticas}

El estudio fue aprobado por el Comité de Ética de la Institución Superior con Acta \# 4,1.01.03.03.20. La población objeto de estudio fueron los jefes directos de los graduados, los cuales fueron tomados de la base de datos de egresados del programa de Fisioterapia del año 2003 al 2017. La participación fue voluntaria con su respectivo consentimiento informado.

\section{Conflicto de Interés}

Los autores de este estudio, expresan que no existe ningún conflicto de interés.

\section{Referencias}

1. ArciniegasCM,BedoyaDP,UrbinaLM.Competencia creatividad e innovación: conceptualización y abordaje en la educación. Katharsis. 2013; 15: 195214. doi: $10.25057 / 25005731.245$.

2. Gómez EP, Agredo VS, David JA. Perfil profesional y ocupacional de los fisioterapeutas en Colombia. CES Mov Salud. 2015; 3(1): 35-43.

3. Zapata WA. Formación por competencias en educación superior. Una aproximación conceptual a propósito del caso colombiano. Rev Iberoam Edu. 2005; 36(9): 1.

4. Grosman PL. Diseño y ajuste de programas de formación para el trabajo bajo el enfoque de competencias. Rev Edu Colombia Ap. 2008; 16.

5. Reimers F, Carnoy M, Brunner J, Panneflek A, Marchesi Á, Namo G, et al. Protagonismo docente en el cambio educativo. Chile AMF Imprenta. 2005; 191.

6. Asociación Colombiana de Facultades de Fisioterapia (ASCOFAFI), Colegio Colombiano de Fisioterapeutas (COLFI) y Asociación Colombiana de Estudiantes de Fisioterapia (ACEFIT). Perfil profesional y competencias del fisioterapeuta en Colombia. Bogotá: Ministerio de Salud y Protección Social. 2015; 30-40.

7. Beneitone P. Reflexiones y perspectivas de la educación superior en América Latina: informe final Proyecto Tuning América Latina: 2004-2007.

8. Cabeza L, Castrillón J, Lombana J. Importancia y coincidencia de competencias para egresados de administración y empleadores: un enfoque por regiones de Colombia. Rev Fac Cienc Econ. Invest Reflex. 2017; 25(2): 105-122. doi: 10.18359/rfce.1983.

9. Cabrera N, López L, Portillo M. Las competencias de los graduados y su evaluación desde la perspectiva de los empleadores. Estud Pedag. 2016; 42(3): 6987. doi: $10.4067 /$ S0718-07052016000400004.

10. AQU.Agència per a la Qualitat del Sistema Universitari de Catalunya. Empleabilidad y competencias de los recién graduados la opinión de empresas e instituciones. B 13431. Primera edición. 2015.

11. Aurelio VS, Poblete M. Capítulo, I. Aprendizaje basado en competencias: una propuesta para la evaluación de las competencias genéricas. Ed Mensajero, Bilbao. 2007. doi:10.15581/004.16.197.

12. Muñoz MN, Rodríguez P, Hincapié J, Agudelo AM, Ramírez R. Percepción de empleadores sobre las competencias de graduados del Programa de Enfermería de la Fundación Universitaria del Área Andina de Pereira (Colombia). Cult Cuidado. 2012; 9(2): 22-38.

13. Peterson JA, Sánchez D. Percepción del empleador: competencias del egresado de Enfermería de la Universidad Popular del Cesar. 2016; 13(26). doi: 10.18041/0124-0102/advocatus.26.944.

14. Bernal CI. Diseño curricular basado en competencias profesionales: una propuesta desde la psicología interconductual. Rev Edu Desarrollo. 2007; 6(2): 45-54.

15. Dussán C, Ruíz FJ, Tamayo ÓE, Montoya M. Perspectiva de los empleadores sobre la pertinencia académica de los programas de pregrado de la universidad de Caldas. Rev Latinoam Est Edu. 2017; 13(2): 30-48. doi: 10.17151/rlee.2017.13.2.3.

16. Herrera EV, Camargo DM. Perfil profesional de los fisioterapeutas egresados de la UIS: 1980-2003. Rev Univ Ind Santander Salud. 2004; 36(1): 2-11. 